OPEN ACCESS

Edited by:

Iñigo Muxika,

Technological Center Expert in Marine and Food Innovation (AZTI), Spain

Reviewed by:

Martin Vohnik,

Institute of Botany (ASCR), Czechia Kevin Alexander Hovel,

San Diego State University, United States

*Correspondence:

Adriana Alagna

adriana.alagna@ias.cnr.it

${ }^{\dagger}$ These authors share first authorship

Specialty section: This article was submitted to Marine Ecosystem Ecology, a section of the journal Frontiers in Marine Science

Received: 02 October 2021 Accepted: 03 December 2021

Published: 06 January 2022

Citation:

Zenone A, Badalamenti F, Alagna A, Gorb SN and Infantes $E$ (2022) Assessing Tolerance to

the Hydrodynamic Exposure of Posidonia oceanica Seedlings

Anchored to Rocky Substrates.

Front. Mar. Sci. 8:788448. doi: 10.3389/fmars.2021.788448

\section{Assessing Tolerance to the Hydrodynamic Exposure of Posidonia oceanica Seedlings Anchored to Rocky Substrates}

\author{
Arturo Zenone ${ }^{1,2,3 \dagger}$, Fabio Badalamenti ${ }^{2,3,4 t}$, Adriana Alagna ${ }^{2 *}$, Stanislav N. Gorb ${ }^{1}$ and \\ Eduardo Infantes 5,6 \\ ${ }^{1}$ Department of Functional Morphology and Biomechanics, Zoological Institute, Kiel University, Kiel, Germany, ${ }^{2}$ Institute \\ for the Study of Anthropic Impact and Sustainability in the Marine Environment, National Research Council, Palermo, Italy, \\ ${ }^{3}$ Department of Integrative Marine Ecology, Sicily Marine Center, Anton Dohrn Zoological Station, Palermo, Italy, ${ }^{4}$ School \\ of Geosciences, Grant Institute, University of Edinburgh, Edinburgh, United Kingdom, ${ }^{5}$ Department of Marine Sciences, \\ University of Gothenburg, Gothenburg, Sweden, ${ }^{6}$ Norwegian Institute for Water Research (NIVA), Oslo, Norway
}

Among a suite of abiotic and biotic factors, the hydrodynamic regime strongly influences the success of seagrass recruitment through sexual propagules. Uprooting of propagules by drag forces exerted by currents and waves is one of the main causes for the failed establishment and the consequent recruitment. Substrate type and stability play a key role in determining the success of colonization through sexual propagules, as seedling establishment probabilities proved to be significantly higher on rocky bottoms than on unstable unconsolidated substrates. In this research, the current and wave flow intensity that Posidonia oceanica seedlings anchored to rocky substrates can withstand before uprooting were evaluated and the influence of substrate complexity on seedling anchorage success and anchorage strength was investigated. P. oceanica seedlings withstood the current velocity of $70 \mathrm{~cm} \mathrm{~s}^{-1}$ and increased orbital flow velocities up to $25 \mathrm{~cm} \mathrm{~s}^{-1}$. Seedling adhesion strength ranged from 3.92 to $29.42 \mathrm{~N}$. Results of the present study corroborate the hypothesis that substrate complexity at scales relevant to the size of propagules is a crucial feature for $P$. oceanica seedling establishment. The intensity of unidirectional and oscillatory flow that seedlings can withstand without being dislodged assessed in this study support the hypothesis that $P$. oceanica sexual propagules, once adhered to a consolidated substrate, are able to tolerate high hydrodynamic stress. The results of the present study contribute to re-evaluation of the habitat requirements of $P$. oceanica, assessing the range of hydrodynamic conditions that this species can tolerate during the early stages of its life history.

Keywords: Posidonia oceanica, resistance, anchorage strength, recruitment, rocky substrate

\section{INTRODUCTION}

Spatial distribution and abundance of seagrasses are controlled by a suite of abiotic and biotic factors (Roca et al., 2016). The knowledge of species' habitat requirements is paramount for establishment of reference conditions for seagrasses presence and survival, for the correct management of the meadows, as well as for the support of restoration strategies (Montefalcone, 2009; Boudouresque et al., 2012). 
Posidonia oceanica (L.) Delile is the most important and endemic Mediterranean seagrass, which has faced an alarming decline of its meadows' extension over the last few decades (Telesca et al., 2015). Gaining a deeper insight into environmental conditions conducive to meadow survival and recovery after disturbance is crucial for the conservation of this valuable habitat.

Traditionally, seagrass ecophysiology has focused on identifying light requirements for meadow persistence, and only recently, the hydrodynamic regime that seagrasses can tolerate has received attention (Koch, 2001; Infantes et al., 2009; Vacchi et al., 2010, 2014). Hydrodynamic stress induced by water movements on submersed aquatic vegetation is thought to set the upper depth limit of meadow distribution (Koch et al., 2006; Infantes et al., 2009; Vacchi et al., 2010), but it can also determine the morphology of $P$. oceanica beds (Buia et al., 2004; Lasagna et al., 2011).

Recently, substrate type has emerged as a key habitat feature, influencing the hydrodynamic disturbance that $P$. oceanica can tolerate and hence determining the depth of the meadow upper limit (Infantes et al., 2009; Montefalcone et al., 2016; Ruju et al., 2018). The models aimed at predicting the upper depth limit of $P$. oceanica were developed on meadows growing on sand and, as such, were not able to predict the landward limit observed on rocky bottoms. Indeed, $P$. oceanica showed to withstand significantly higher hydrodynamic forcing when settled on rock than on soft bottoms (Montefalcone et al., 2016; Ruju et al., 2018). This pattern was generally ascribed to the potential of the rocky substrate to offer a firm anchorage compared to sand and to the plasticity of the plant root system to adapt to different substrate types (Montefalcone et al., 2016; Ruju et al., 2018).

The hydrodynamic regime not only affects the spatial distribution and arrangement of established seagrass beds but also strongly influences the success of seagrass recruitment through sexual propagules. Uprooting of propagules by drag forces exerted by currents and waves and subsequent displacement to unsuitable sites is one of the main causes for the failed establishment and low survival of seagrass early life history phases (Olesen et al., 2004; Irving et al., 2010; Rivers et al., 2011; Marion and Orth, 2012). Indeed, the mature meadows tolerate higher drag forces than early life phases as mature plants possess a network of rhizome and roots that firmly anchor them to the substrate, while sexual propagules are provided with a limited rooting system and thus are more subjected to displacement by water movements (Koch, 2001; Infantes et al., 2011).

Notably, the majority of the sexual recruitment sites reported in the literature were located at a depth shallower than those of the adjacent meadows, in areas where hydrodynamic stress generated by waves is reasonably higher than that experienced by the mature, deeper meadows.

The ability of seedlings and young plantlets to withstand hydrodynamic forces produced by waves and currents is determined by the strength of their anchorage to the substrate via roots (Infantes et al., 2011; Rivers et al., 2011). Recently, the adhesive properties of root hairs in $P$. oceanica seedlings have been documented (Alagna et al., 2015, 2020; Badalamenti et al., 2015; Borovec and Vohník, 2018; Guerrero-Meseguer et al., 2018; Zenone et al., 2020a,b). This morpho-physiological trait has been shown to provide an adaptive advantage for early seedling establishment on rocky bottoms. In fact, when reared on consolidated substrates, seedlings reached a strong anchorage via root hair adhesion in a few weeks, while they failed to establish on sand (Alagna et al., 2015).

However, Alagna et al. (2015) provided only an indirect, qualitative estimation of the wave energy that seedlings attached on rocks can withstand before being uprooted. Quantitative measures of the hydrodynamic drag forces in terms of wave and current-induced velocities that seedlings anchored to rocks can tolerate are lacking.

The main objectives of this research were: (i) to quantitatively evaluate the current and wave flow intensity that $P$. oceanica seedlings anchored to rocky substrates can withstand before uprooting and (ii) to investigate the influence of substrate complexity at the scale of $\mathrm{cm}$ and $\mathrm{mm}$ on seedling anchorage success and anchorage strength. Our hypothesis was that proper plant-substrate interaction can only take place if the size of grooves, pits, or other substrate irregularities is similar to the size of the plant organs that play a role in propagule dispersal and settlement (i.e., the size of the seed and root and root hair). This interaction might allow the seedling dispersed by water movements (a) to become persistently trapped in centimeterswide asperities, (b) root hairs to grow if and when micron-wide asperities are present, according to the well-studied adhesion mechanism described in Zenone et al. (2020a,b), and (c) roots to grow inside millimeters-wide irregularities (i.e., substrate grooves) by increasing anchorage strength due to entanglement and friction. Two tests of resistance were performed on seedlings grown on three levels of substrate complexity by (i) exposing seedlings to increasing current and wave flows and (ii) measuring the pulling forces needed to dislodge seedlings. The main predictions were that seedlings grown on substrates with higher complexity would have a higher anchorage rate and higher anchorage strength, offering a greater resistance in all the hydrodynamic and pulling tests.

\section{MATERIALS AND METHODS}

\section{Seed Collection}

Beach casted fruits of $P$. oceanica were collected between the 24th of April and the 5th of May 2016, in the Gulf of Carini, along the NW Sicilian coast. Seeds were immediately removed from fruits and stored in a refrigerated box filled with seawater for transport. Seeds $(n=875)$ were transported to Kristineberg Marine Research Station in Sweden, by the end of May 2016. Three tanks of 1,000 $\mathrm{L}(1.5 \times 1.5 \mathrm{~m})$ were set up with similar conditions, i.e., salinity $38 \mathrm{ppt}$, temperature of $20^{\circ} \mathrm{C}$, light of $80 \mu$ moles $\mathrm{m}^{-2} \mathrm{~s}^{-1}$, and a 12/12 h light/dark photoperiod.

\section{Seedlings Survival and Anchorage}

To analyze the effects of substrate complexity on seedling anchorage, seedlings were grown for 4 months on $10 \times$ $10 \mathrm{~cm}$ calcareous tiles characterized by three different levels of complexity: (i) Low complexity (LC), tiles provided with complexity only at the $\mu m$ scale, i.e., the natural roughness 


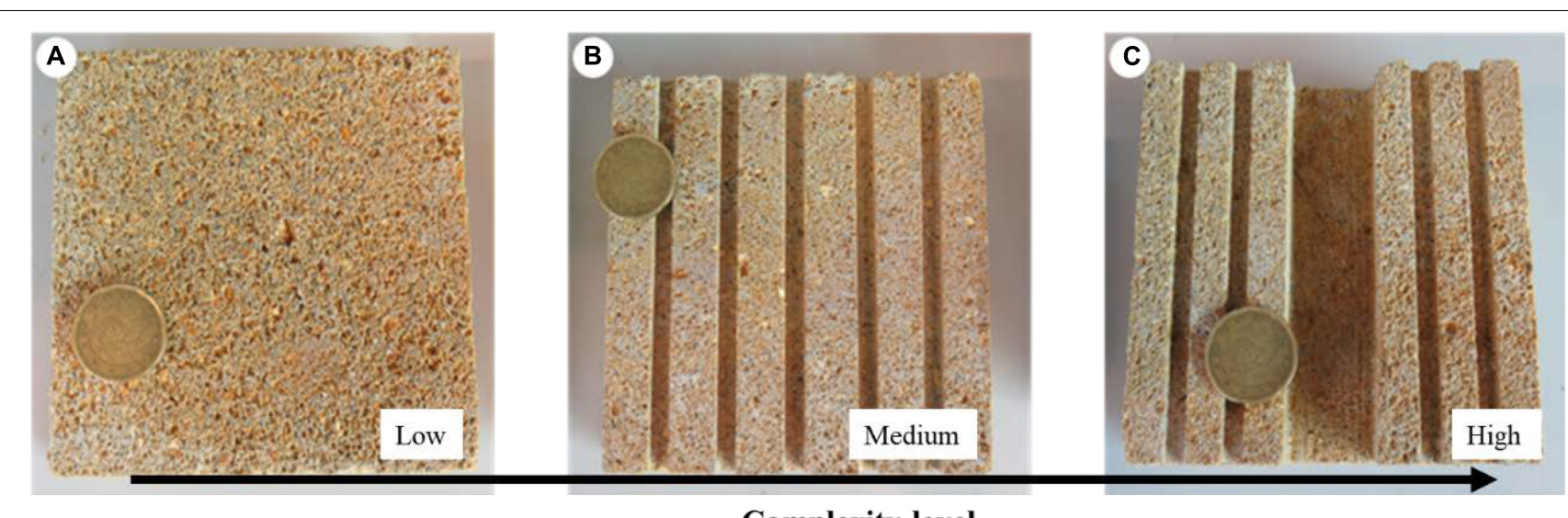

Complexity level

FIGURE 1 | Calcareous tiles characterized by three levels of complexity on which the seedlings were grown. (A) Low complexity, (B) medium complexity with five linear crevices $0.4 \times 0.4 \mathrm{~cm}$, and $(\mathbf{C})$ high complexity with four linear crevices of $0.4 \times 0.4 \mathrm{~cm}$ and a slot of $2 \times 1 \mathrm{~cm}$. The diameter of the coin is $22.25 \mathrm{~mm}$.

of the stone; (ii) Medium complexity $(M C)$, tiles provided with complexity at the $\mu \mathrm{m}$ and $\mathrm{mm}$ scale, having five parallel linear crevices $(0.4 \times 0.4 \mathrm{~cm})$, and (iii) High complexity $(H C)$, tiles provided with complexity at the $\mu \mathrm{m}, \mathrm{mm}$, and $\mathrm{cm}$ scale, having a central slot $(2 \times 1 \mathrm{~cm})$ and four parallel crevices $(0.4 \times 0.4 \mathrm{~cm})$ (Figure 1). One seedling was laid down at the center of each tile, each representing an independent replicate, and randomly allocated to one of the three culture tanks, for an overall number of 240 seedlings. At the end of the experiment, the number of living seedlings and the number of seedlings anchored to the tiles were counted. A seedling was considered anchored to the substrate when trying to gently move it by hand it opposed a clear resistance; otherwise, it was considered not anchored. Growth performances were evaluated through seedling morphological and biomass variables determined on 10 replicates for each complexity level. Number of standing leaves (Nle), maximum leaf length (Mll), maximum leaf width (Mlw), total leaf area per seedling (Tla, i.e., the sum of the area of all standing leaves), number of root $(\mathrm{Nr})$ and of root branches $(\mathrm{Nb})$, total root length (Trl, i.e., the sum of the length of all the roots), average root width ( $\mathrm{Rw}$ ), number of adhered root (N.ar), and root adhesion length (Ral, i.e., the sum of the length of all the portions of the seedling roots attached to the substrate) were measured. The assessed biomass variables, consisted of seedling total biomass (Tdw) and the relative percentage contribution of seed, roots, and leaves (Sdw; Rdw; Ldw) to Tdw, were measured as dry weight after oven-drying at $60^{\circ} \mathrm{C}$ until a constant weight was reached (precision $0.0001 \mathrm{~g}$ ). All measures were reported as centimeters $(\mathrm{cm})$ and grams $(\mathrm{g})$.

\section{Seedling Tolerance to the Hydrodynamic Exposure}

The maximum current and wave intensity tolerated by seedlings anchored to the tiles was assessed by exposing them to unidirectional (current) and oscillatory (waves) flow. Tolerance to hydrodynamic forces was expressed as the threshold velocities $\left(u, \mathrm{~cm} \mathrm{~s}^{-1}\right)$ needed to dislodge seedlings from the tiles, which was measured using two hydraulic flumes located at Kristineberg Marine Research Station, Sweden. Since no seedlings were anchored on LC, measurements were performed only on HC and MC. The number of replicates depended on the availability of anchored seedlings.

The current flume was $4 \mathrm{~m}$ long, $0.5 \mathrm{~m}$ wide, and $0.5 \mathrm{~m}$ depth, had a $2 \mathrm{~m}$ long test section with a water level of $0.2 \mathrm{~m}$. Flow velocities were generated by a motor-run propeller located at the far end of the flume controlled by an adjustable speed drive (Dayton Electronic, 6K119). Seedlings were exposed to a unidirectional bottom current of $70 \mathrm{~cm} \mathrm{~s}^{-1}$ for $40 \mathrm{~min}$, a speed reached by gently increasing the flume current velocity within $10 \mathrm{~min}$ to the maximum unidirectional flow velocity the flume could produce ( $2 \mathrm{~min}$ at each of the following velocities 0,5 , 10,35 , and $50 \mathrm{~cm} \mathrm{~s}^{-1}$ ). The wave flume was $3.5 \mathrm{~m}$ long, $0.6 \mathrm{~m}$ wide, and $0.8 \mathrm{~m}$ deep, with PVC modules placed in the bottom with a $35 \times 35 \mathrm{~cm}$ box for holding the tiles with the seedlings anchored on them. The flume was driven by a pneumatic pedal that generated waves by adjusting the piston stroke and speed (see flume details in Infantes et al., 2021). A wave absorber made of synthetic fibers with a slope of $20^{\circ}$ was placed at the end of the flume to reduce wave reflections. Water depth was $0.25 \mathrm{~m}$. Seedlings were exposed to four increasing orbital flow velocities of 5,10 , and $20 \mathrm{~cm} \mathrm{~s}^{-1}$ for $2 \mathrm{~h}$ each, to reach the maximum orbital flow velocity the flume could generate of $25 \mathrm{~cm} \mathrm{~s}^{-1}$, for $18 \mathrm{~h}$. Flow velocities in both flumes were measured with an acoustic Doppler velocimeter, ADV (Nortek, Vectrino) with a sampling rate of $25 \mathrm{~Hz}$, sampling volume of $7 \mathrm{~mm}$, and a nominal velocity range of $0.3 \mathrm{~m} / \mathrm{s}$. Measurements were performed at $10 \mathrm{~cm}$ above the bottom and $5 \mathrm{~cm}$ in front of the sample to not interact with the leaves (Figure 2).

\section{Seedling Anchorage Strength and Uprooting Resistance}

The force needed to detach seedlings from the substrate $(F)$ was measured on an independent set of individuals and used as a proxy for seedling anchorage strength. A hook-shaped 


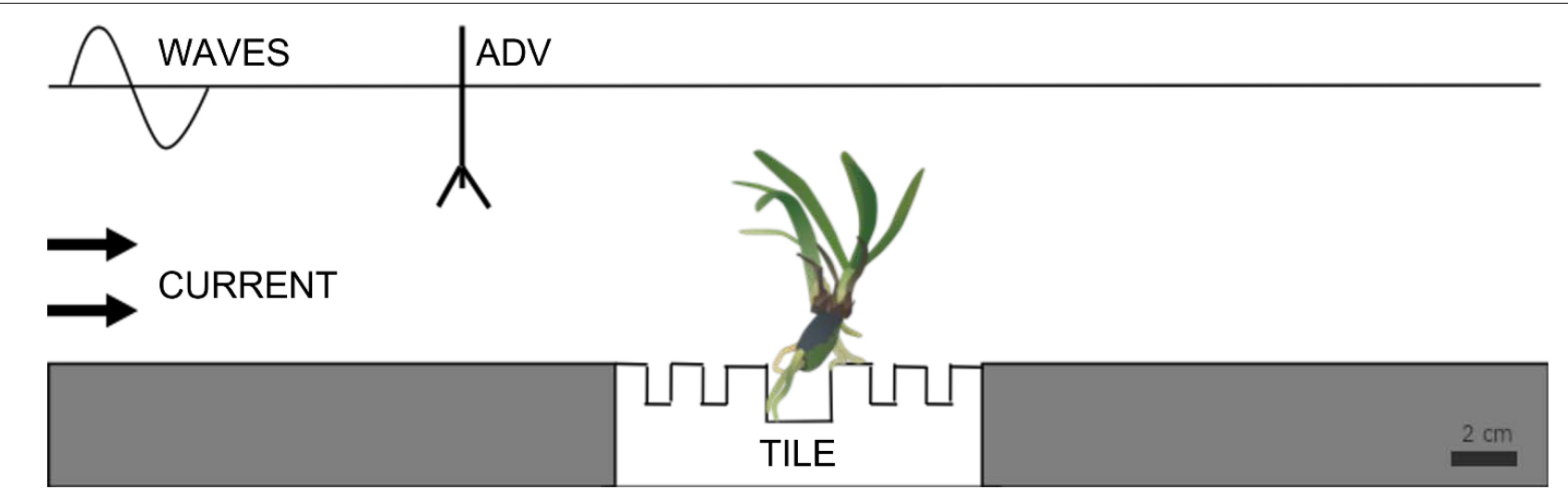

FIGURE 2 | Schematic representation of the experimental setup to test the resistance of root adhesion to the substrate in the current and wave flumes (ADV, acoustic Doppler velocimeter).

plastic-coated iron wire, $3 \mathrm{~mm}$ in diameter, was carefully passed underneath the seed body. One end of the wire was connected to a digital spring scale (Vetek, precision $5 \mathrm{~g}$ ). Measurements were taken by pulling the seedling perpendicular to the substrate until detachment. The pull-off weight was converted to force $(\mathrm{N})$. Measures were performed only for $\mathrm{HC}$ and MC complexity levels as seedlings placed on LC tiles did not show anchorage.

Based on the Kendall peeling model, according to which the adhesion force in biological systems is proportional to the peeling line (i.e., the sum of the width of all the adhesive elements participating in the attachment process) (Varenberg et al., 2010), the root adhesion capacity (Rac) was defined as:

$$
R A C=N \cdot a r^{*} R w
$$

where N. ar is the number of roots adhered to the tile, and $R w$ is the average width of the root $(\mathrm{cm})$.

The maximum flow velocity that anchored seedlings can potentially withstand before being uprooted was estimated using the equation:

$$
u=\sqrt[2]{2 F d / C_{D} \rho a}
$$

(rearranged from Dean and Dalrymple, 1991), where $u$ is the flow velocity $\left(\mathrm{m} \mathrm{s}^{-1}\right), F_{d}$ is the force acting over an individual plant measured as the force $(\mathrm{N})$ required to detach seedlings from the tiles ( $F$, pulling test); $C_{D}$ is the drag coefficient calculated as 1.2 for 25-week-old P. oceanica seedlings (Pereda-Briones et al., 2018); $\rho$ is seawater density, set at $1,025 \mathrm{~kg} \mathrm{~m}^{-3}$; and $a$ is the total leaf area per seedling $\left(\mathrm{m}^{2}\right)$.

\section{Statistical Analysis}

Chi-squared was used to detect significant differences in the number of anchored individuals between complexity levels $(n=80)$. Seedling survival and anchorage rates were computed on each of the three tanks. The effect of substrate complexity on the flow velocity needed to dislodge the seedlings from the tiles and on seedling anchorage strength (pulling test) $(n=10)$ was analyzed by a one-way analysis of variance (ANOVA), with factor Complexity (Co), fixed, two levels, namely, MC and HC, as seedlings did not anchor on LC tiles. The same experimental design was applied to test for the effects of substrate complexity on variables used to characterize the plant biometry (Nle, Mll, Mlw, Tla, Trl, Rw, Nr, Nb, and Ral) and the biomass allocation (Tdw, Sdw, Ldw, and Rdw) except for the factor Co that had three levels instead of two, namely, LC, MC, and HC.

Before running the analysis, the homogeneity of variances was tested using Cochran's C test. Student-Newman-Keuls (SNK) tests allowed the appropriate means comparison between levels, when the effect of a treatment factor was significant $(P<0.05)$. The relation between the relevant root morphological variables (Rw, Ral, and $\mathrm{Rac}$ ) and the anchorage strength ( $F$, pulling test) was tested with a simple linear regression model. Finally, the same analysis was employed to test the relation between the root adhesion capacity (Rac) and the maximum flow velocity tolerated by seedlings before detachment $(u)$, calculated with Eq. 1 .

\section{RESULTS}

\section{Seedlings Survival and Anchorage}

After 4 months of indoor culturing, seedling survival rate was high, with an overall mean of $0.97 \pm 0.05$ (mean \pm SE). Seedling anchorage rate significantly varied according to complexity levels. Anchorage rate was significantly higher (chi-square $=10.27$; $P=0.001)$ in the high complexity $(\mathrm{HC})(0.55 \pm 0.03)$ than in the medium complexity level (MC) $(0.29 \pm 0.01)$. None of the seedlings in the low complexity level (LC) anchored to the substrate. Seedling anchorage occurred through the production of hairs in the piliferous zone of the adventitious roots that allowed adhesion to the tile surface.

At the end of the experiment, seedlings were 5 months old and had on average $7.00 \pm 0.27$ leaves (mean \pm SE) (Table 1). The seedling shoots reached an average total leaf area of $28.92 \pm 1.64$ $\mathrm{cm}^{2}$, showing a maximum leaf length and a maximum leaf width of $10.41 \pm 0.34 \mathrm{~cm}$ and of $0.65 \pm 0.01 \mathrm{~cm}$, respectively, with no difference between complexity levels [Nle $F_{(2,27)}=0.13$, $P=0.881 ; \operatorname{Mll} F_{(2,27)}=0.59, P=0.564$; Mlw $F_{(2,27)}=1.66$, $P=0.209$; Tla $\left.F_{(2,27)}=0.06, P=0.945\right]$. Root architecture varied across complexity levels. Total root length ranged from 
TABLE 1 | Seedling morphological and biomass variables.

\begin{tabular}{lcccc}
\hline Complexity & Nle (n) & Tla (mmq) & MIl (mm) & Mlw (mm) \\
\hline Leaves & & & & \\
HC & $7.1 \pm 0.3$ & $2970.86 \pm 300.61$ & $108.95 \pm 7.19$ & $6.28 \pm 0.22$ \\
MC & $7.1 \pm 0.6$ & $2835.91 \pm 295.31$ & $99.74 \pm 5.76$ & $6.73 \pm 0.12$ \\
LC & $6.8 \pm 0.4$ & $2869.13 \pm 287.74$ & $103.68 \pm 4.94$ & $6.42 \pm 0.19$ \\
\hline Complexity & Trl (cm) & Rw (cm) & Nb (n) & Ral (cm) \\
\hline Roots & & & & \\
HC & $33.01 \pm 3.38$ & $0.15 \pm 0.01$ & $5.7 \pm 2.6$ & $10.69 \pm 2.74$ \\
MC & $21.47 \pm 2.22$ & $0.16 \pm 0.01$ & $0.8 \pm 0.4$ & $3.69 \pm 2.26$ \\
LC & $30.50 \pm 2.08$ & $0.15 \pm 0.01$ & $0.3 \pm 0.3$ & 0.00 \\
\hline Complexity & Tdw (g) & Sdw (g) & Ldw (g) & Rdw (g) \\
\hline Biomass & & & & \\
HC & $0.60 \pm 0.03$ & $0.27 \pm 0.02$ & $0.13 \pm 0.01$ & $0.20 \pm 0.02$ \\
MC & $0.52 \pm 0.04$ & $0.29 \pm 0.02$ & $0.11 \pm 0.01$ & $0.15 \pm 0.01$ \\
LC & $0.52 \pm 0.03$ & $0.26 \pm 0.02$ & $0.11 \pm 0.01$ & $0.15 \pm 0.01$
\end{tabular}

Mean values \pm standard errors. HC, high complexity; MC, medium complexity; LC, low complexity; Nle, number of standing leaves; Tla, total leaf area per seedling; MII, maximum leaf length; MIW maximum leaf width; Trl, total root length; Rw, root width; Nb, number of root branches; Ral, root adhesion length; Tdw, seedling total biomass; Sdw, seed biomass; $L d w$, leaf biomass and Rdw, root biomass.

$21.47 \pm 2.22 \mathrm{~cm}$ in $\mathrm{MC}$ tiles to $33.01 \pm 3.38 \mathrm{~cm}$ in $\mathrm{HC}$ tiles and was significantly lower in MC compared to $\mathrm{HC}$ and LC levels, which did not differ from each other $\left[F_{(2,27)}=5.34, P=0.011\right]$ (Table 1). Root width was on average $0.150 \pm 0.004 \mathrm{~cm}$, with no differences between complexity levels $\left[F_{(2,27)}=1.38, P=0.268\right]$. Roots resulted significantly more branched $\left[F_{(2,27)}=3.87\right.$, $P=0.033]$ in $\mathrm{HC}(5.70 \pm 2.61$ branches $)$, than in $\mathrm{MC}(0.80 \pm 0.42)$ and LC complexity levels $(0.30 \pm 0.30)$ that did not differ from each other. None of the seedlings grown on LC complexity level adhered to the substrate while the root adhesion extent was significantly higher in seedlings grown on $\mathrm{HC}(10.69 \pm 2.74 \mathrm{~cm})$, than in those grown on $\mathrm{MC}(3.69 \pm 2.26)\left[F_{(2,27)}=7.01\right.$, $P=0.004$ ] (Figure 3). At the end of the experiment, seedling total biomass reached on average $0.55 \pm 0.02 \mathrm{~g}$, with no differences across complexity levels $\left[F_{(2,27)}=1.63, P=0.215\right]$ (Table 1). Similarly, there was no difference in seed and leaf biomass between complexity levels [Sdw $F_{(2,27)}=0.47, P=0.629$; Ldw $\left.F_{(2,27)}=0.13, P=0.880\right]$, with a mean value of $0.27 \pm 0.01 \mathrm{~g}$ and $0.12 \pm 0.01 \mathrm{~g}$, respectively, while root biomass was significantly higher $\left[F_{(2,27)}=5.21, P=0.012\right]$ in seedlings grown on $\mathrm{HC}$ complexity tiles $(0.20 \pm 0.02 \mathrm{~g})$ compared to seedlings grown on the $\mathrm{MC}(0.15 \pm 0.01 \mathrm{~g})$ and $\mathrm{LC}(0.15 \pm 0.01)$ complexity tiles, which did not differ from each other (Figure 3 ).

\section{Seedling Tolerance to the Hydrodynamic Exposure}

All seedlings exposed to currents ( $\mathrm{HC}$ and $\mathrm{MC}$ complexity level) withstood the velocity of $70 \mathrm{~cm} \mathrm{~s}^{-1}$, during the $40 \mathrm{~min}$ exposure. All seedlings exposed to waves (HC and MC complexity level) withstood increased orbital flow velocities up to $25 \mathrm{~cm} \mathrm{~s}^{-1}$ during the $24 \mathrm{~h}$ exposure. None of the seedlings or seedling roots detached or were damaged after the unidirectional flow and the wave experiments.

\section{Seedling Anchorage Strength and Estimation of the Seedling Uprooting Resistance}

Seedling adhesion strength on MC and HC complexity levels ranged from 3.92 to $29.42 \mathrm{~N}$. No differences $\left[F_{(1.18)}=0.01\right.$; $p=0.915$ ] were found between complexity levels, reporting an overall mean value of $11.50 \pm 1.44 \mathrm{~N}$

The adhesion strength was found significantly dependent on the number of adhered roots [N. ar, $r=0.74 ; F_{(1.18)}=21.61$; $p<0.001$ ] and on the root adhesion capacity [RAC, $r=0.75$; $\left.F_{(1.18)}=23.32 ; p<0.001\right]$ (Figure 4), while it was not significantly related to the average root width [RW, $r=0.26$; $\left.F_{(1.18)}=1.31 ; p=0.267\right]$ and the root adhesion length [RAL, $\left.r=0.25 ; F_{(1.18)}=1.25, p=0.279\right]$. Seedlings attached to the substrate with a small portion of their root apparatus resulting in a "seedling on stilts" shape (Figure 5). In fact, only one-third $(35.29 \pm 4.72 \%)$ of the total seedling root system was found anchored to the tiles.

Because the highest unidirectional and oscillatory flow velocities that the flumes were able to generate were not sufficient to cause seedling detachment, the magnitude of the velocities that could have caused seedling detachment was estimated using Equation 2. The maximum flow velocity that the anchored seedlings could potentially withstand in the field before being dislodged ranged from 48.6 to $135.5 \mathrm{~cm} \mathrm{~s}^{-1}$, with a mean value of $87.03 \pm 26.39 \mathrm{~cm} \mathrm{~s}^{-1}$. The maximum flow velocity tolerated by seedlings before being uprooted was found significantly related to the root adhesion capacity [RAC, $r=0.46 ; F_{(1.18)}=4.78$; $p=0.042]$ (Figure 4).

\section{DISCUSSION AND CONCLUSION}

This work quantitatively evaluates for the first time the tolerance of $P$. oceanica seedlings anchored to rocky substrates to the hydrodynamic exposure, providing novel insight into the habitat requirements and colonization potential of this species on rocky shores. Moreover, the findings of the present study contribute to identifying microhabitat features that are crucial for $P$. oceanica seedling settlement and establishment, since substrate complexity at the seed and the root size scales proved to strongly affect seedling anchorage success.

Seedling survival exceeded $95 \%$ at the end of the experiment, confirming that $P$. oceanica sexual propagules can be easily cultivated in controlled tanks for several months, once proper environmental conditions are provided (Belzunce et al., 2008; Infantes et al., 2011; Alagna et al., 2015). Levels of topographical complexity of rocky substrates tested in this study strongly affected the success of seedling anchorage through adhesive root hairs. No anchoring of seedlings was recorded on flat tiles. Instead, more than a quarter of seedlings anchored on tiles of medium complexity and more than half anchored on those with high complexity. Recent works have documented the ability of $P$. oceanica young plantlets to firmly anchor to 

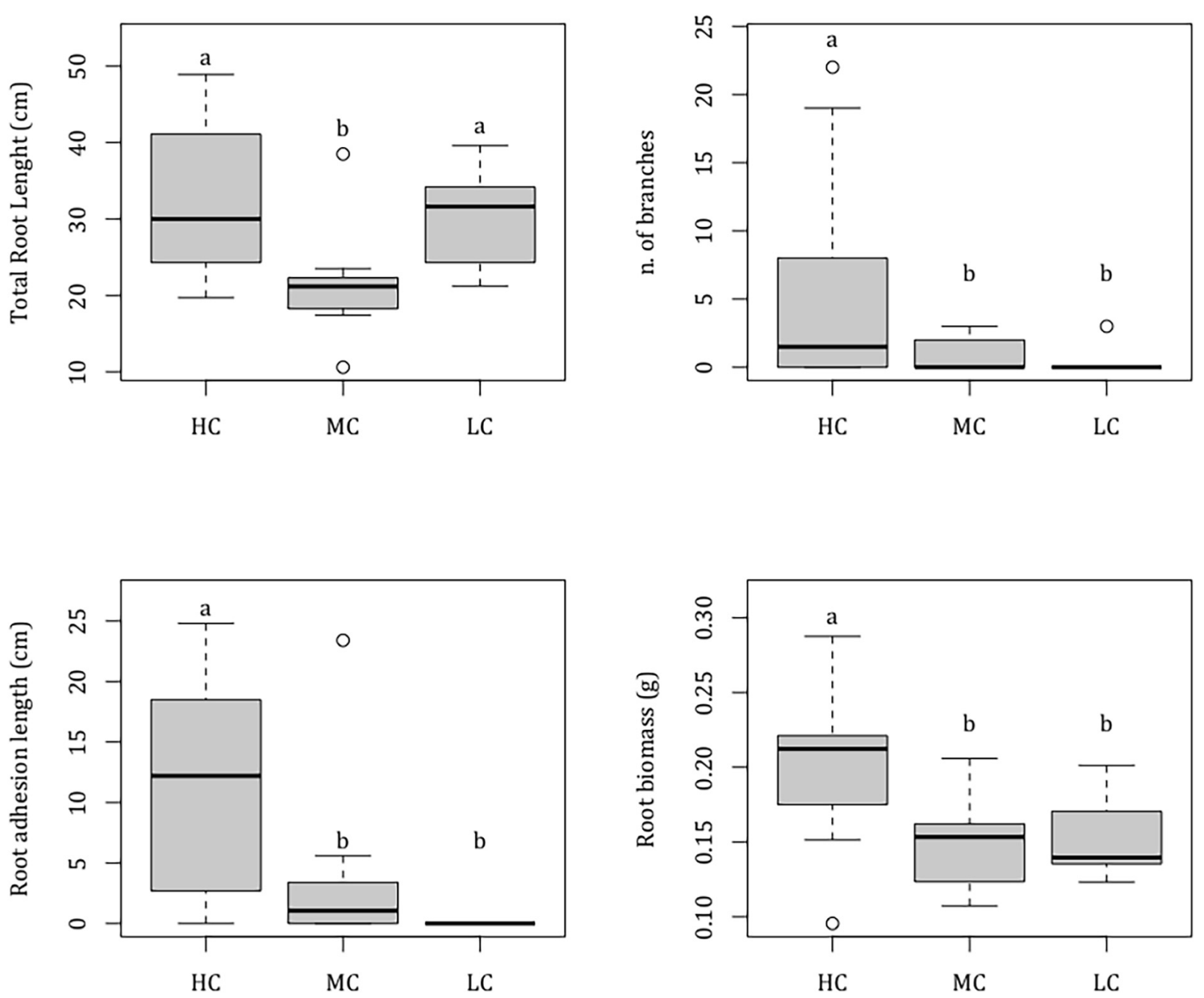

FIGURE 3 | Boxplots showing the effect of substrate complexity (Co) on seedling morphological and biomass variables (total root length, n. of root branches, root adhesion length, and root biomass). Results are showed only for variables for which a significant effect of factor Co was detected. HC = high complexity; $\mathrm{MC}=$ medium complexity; LC $=$ low complexity; Median $=$ horizontal line; $25^{\text {th }}$ and $75^{\text {th }}$ percentiles $=$ vertical boxes; $10^{\text {th }}$ and $90^{\text {th }}$ percentiles $=$ whiskers. $n=10$ for each complexity level. Letters above bars indicate significant differences between experimental groups.

rocks during the first weeks of life due to the presence of root hairs capable of attaching to consolidated substrates and which for this reason have been termed "adhesive" or "sticky" hair (Alagna et al., 2015; Badalamenti et al., 2015). Further research has highlighted that the bioadhesion process is based on a mechanical interlocking mechanism of the root hair tips that grow and adapt to the substrate micro-roughness (Zenone et al., 2020a,b). This process is also supported by the presence of amorphous substances secreted in the contact region (Zenone et al., 2020a). Our findings point out that the availability of consolidated substrates, although provided with roughness at the micrometer scale, does not guarantee alone that root adhesion occurs. Proper levels of complexity at a scale relevant for the seed, i.e., 1-2 cm, for the roots, i.e., few $\mathrm{mm}$ and for the root hairs, i.e., tens of $\mu \mathrm{m}$, are crucial, in order to achieve early seedling anchorage to consolidated substrates. The anchorage process could be summarized as a multi-step mechanism acting on hierarchical spatial scales: the sinking seeds are blocked by the small slots $(\mathrm{cm})$, roots grow in between the interstices $(\mathrm{mm})$ and the root hairs adhere in between the asperities of the substrate $(\mu \mathrm{m})$.

The different levels of substrate complexity tested in the present study showed to affect the architecture of the seedling root system. Seedlings developed a greater root biomass when growing on high complexity tiles due to the greater branching achieved if compared to those of medium and low complexity. The greater branching also involved a greater extension of the roots adhered to the substrate. Seedlings showed overall longer roots when compared to those of similar age grown on a rocky matrix in the study of Alagna et al. (2015) and a greater percentage of biomass was allocated to the roots than leaf compartment, a trait that seems to be in common with that observed for the same species during the natural colonization of rhizomes on consolidated substrates 

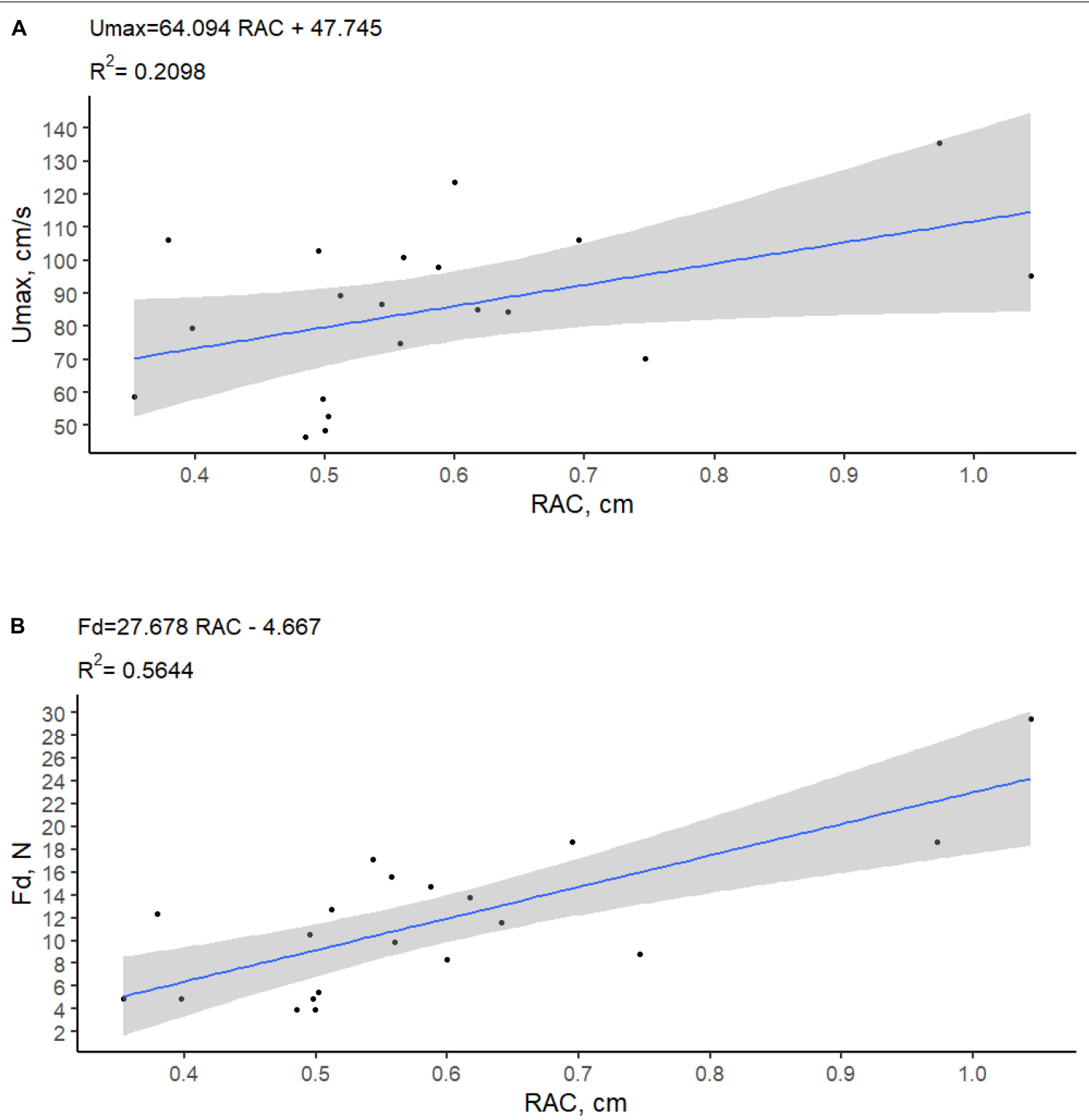

FIGURE 4 | Adhesion strength measured as (A) maximum flow velocity (Umax, $\mathrm{cm} / \mathrm{s})$ and $\mathbf{( B )}$ pulling force $(F d, \mathrm{~N})$ required to detach $P$. oceanica seedlings according to the root adhesion capacity $(R A C, \mathrm{~cm})$.

(Di Carlo et al., 2007). The lack of an optimal complexity at the seed and root scale offered by the tile surface, compared to the rocky matrix used to rear seedlings in Alagna et al. (2015), may have fostered root development in search of a suitable place for adhesion.

The highest seedlings adhesion rate in our study was recorded on high complexity tiles (55\%), a value much lower than that obtained by Alagna et al. (2015) on calcareous rocks (86\% anchorage rate). This difference is most likely due to the different features of the substrate on which seedlings were grown in the two experiments. In the present study, the tiles, although provided with different levels of complexity, offered a much greater amount of sub-horizontal surfaces compared to the rocky matrix used in the study by Alagna et al. (2015) that prevented the roots from developing according to positive gravitropism. In our experiment, once the tip of an adventitious root reached the tile, it mostly faced a flat surface that offered almost no contact between the root hair and the substrate. Over time, the adventitious roots grow longer, lifting the seedling up to several centimeters above the tile surface. The tip of the roots leans on the tile surface without providing any adhesion or it attaches to the substrate only with a small portion above the root cap: the overall result is that of a "seedling on stilts."

The maximum force needed to detach seedlings from the tiles and, consequently, the maximum flow velocity tolerated by seedling before uprooting were significantly related to the root adhesion capacity (the number of adhered roots multiplied by the average root width), while other variables, such as the root adhesion length, did not show significant effects. According to the Kendall peeling model, in biological fibrillar adhesive systems operating at the micrometer and nanometer scale, the force needed to detach an adhesive pad from a flat surface 


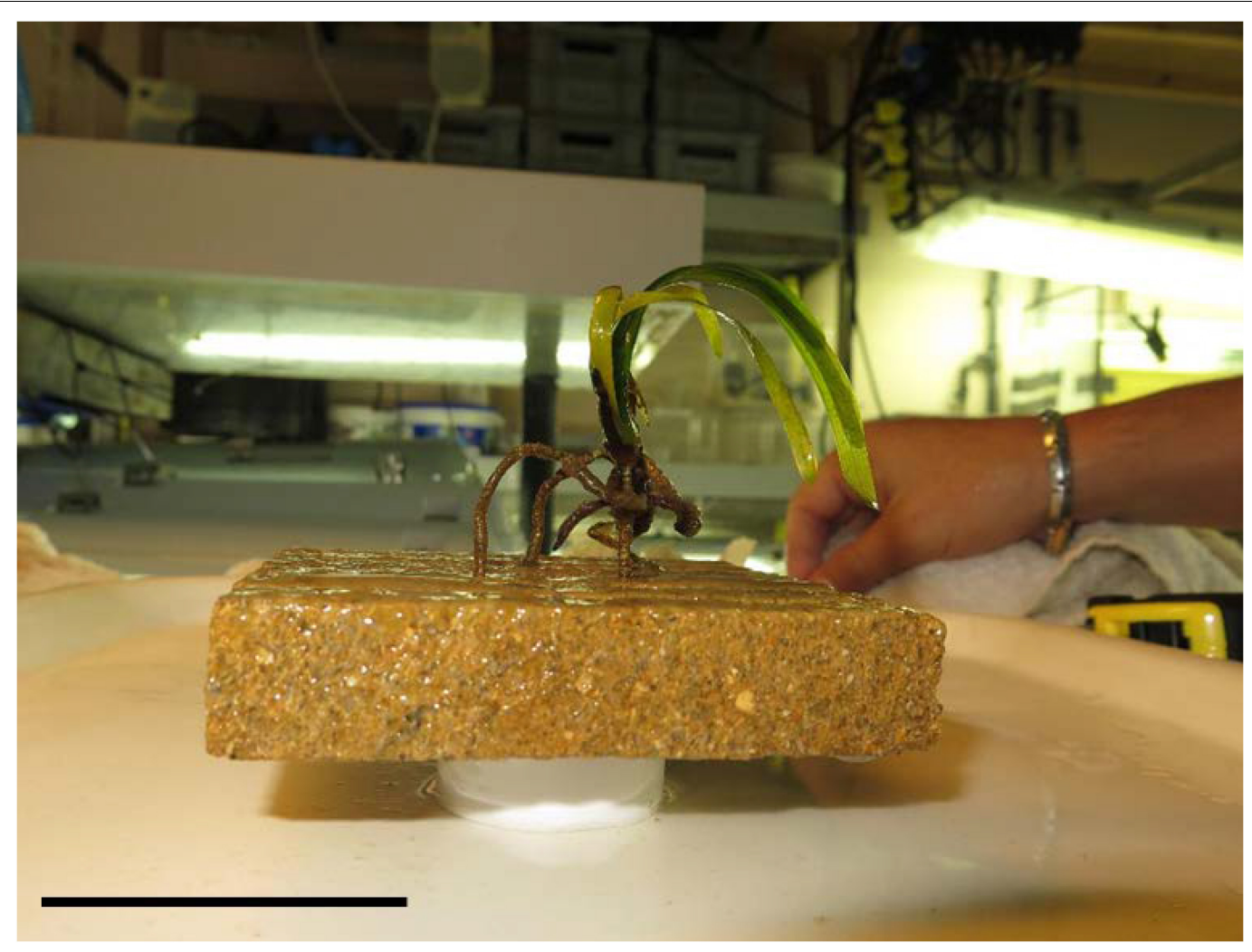

FIGURE 5 | Detail of a "seedling on stilts." Over time, the seedling adventitious roots grew longer, rising the seedling up to several centimeters above the tile surface without causing a significant adhesion. The tip of the roots leans on the plate or attaches to the substrate only with the distal portion. Scale bar, $5 \mathrm{~cm}$.

is proportional to the "peeling line length," i.e., the sum of the width of all the elements participating in the attachment process (Varenberg et al., 2010). Our results suggest that a similar relation could work even at higher spatial scales and on irregular substrates, as root adhesion capacity proved to be a good explanatory variable for seedling adhesion force and performed better than any other variable tested.

Our findings support previous field studies where seagrass seedlings showed higher recruitment probabilities on consolidated and firm substrates provided with topographical complexity (i.e., rocky substrates and dead matte of P. oceanica) than on mobile (i.e., sandy) bottoms (Balestri et al., 1998; Piazzi et al., 1999; Olesen et al., 2004; Rivers et al., 2011; Alagna et al., 2013; Balestri et al., 2017; Pereda-Briones et al., 2020; Castejón-Silvo and Terrados, 2021). However, few studies have tried to disentangle the effect of substrate stability from that of complexity (Alagna et al., 2015; Pereda-Briones et al., 2018). Indeed, sea bottom topographical complexity affects propagule dispersion and retention (Inglis, 2000; Pereda-Briones et al., 2018; Meysick et al., 2019), as shown by the positive correlation found between substrate topographical complexity at the scale of centimeters and propagule trapping (Pereda-Briones et al., 2018). This pattern was ascribed to the increased flow reduction over substrates of higher complexity, which allow for propagule retention (Pereda-Briones et al., 2018). Hole and crevices on rocky substrates as well as inside seagrass dead matte would reduce the hydrodynamic drag forces acting on propagules, favoring their retention and establishment (Olesen et al., 2004; Rivers et al., 2011; Alagna et al., 2013; Pereda-Briones et al., 2018). Results of the present study contribute to disentangling the effects of substrate stability from that of substrate complexity and corroborate the hypothesis that substrate complexity at scales relevant to the size of propagules is a crucial feature for $P$. oceanica seedling establishment.

The maximum intensity of unidirectional and oscillatory flow that seedlings can withstand without being dislodged, measured in the present study, supports the hypothesis that $P$. oceanica sexual propagules, once adhered to a consolidated substrate, are able to tolerate significantly higher hydrodynamic stress compared to seedlings established on sand. In fact, no seedling detached from the tiles under both the unidirectional $(70 \mathrm{~cm}$ $\left.\mathrm{s}^{-1}\right)$ and oscillatory flow $\left(25 \mathrm{~cm} \mathrm{~s}^{-1}\right)$ while, in a previous study, seedlings growing on sand did not persist above $18 \mathrm{~cm}$ $\mathrm{s}^{-1}$ wave-induced velocities (Infantes et al., 2011). Moreover, the estimated maximum orbital velocities that seedling could potentially tolerate in the field before being uprooted suggest that $P$. oceanica sexual propagules settled on rocky bottoms are able to persist at sites characterized by strong wave exposure, up to $87.03 \mathrm{~cm} \mathrm{~s}^{-1}$ orbital velocities, on average, and that some individuals can tolerate peak of $135.5 \mathrm{~cm} \mathrm{~s}^{-1}$ orbital velocities. These wave-induced velocities are comparable or even higher than those estimated to impinge on the upper limit distribution 
of $P$. oceanica meadows found on rocky shores at very shallow sites (depth $<1 \mathrm{~m}$, Ruju et al., 2018).

The presence of $P$. oceanica meadows at locations characterized by strong hydrodynamic exposure can be the result of stepwise clonal growth from already established patches at higher depths or of a direct sexual recruitment and patch initiation from seedlings. Results reported here show how $P$. oceanica sexual propagules can persist and potentially colonize rocky bottoms in high-energy habitats thanks to the adhesive properties of seedling root hairs. The fast and strong anchorage to consolidated substrates provided by this adaptive trait could explain why this species is found at very shallow sites up to the outer surf zone of storms on hard bottoms while it is absent on the same environmental conditions on mobile substrates or on erodible rocks (Montefalcone et al., 2016; Ruju et al., 2018).

The maximum seedling tolerance to wave-induced hydrodynamic stress here estimated represents a new threshold for $P$. oceanica colonization and survival via sexual propagules. Furthermore, this result provides an explanation for the numerous records of successful seedling recruitment at sites shallower than the upper limit distribution of adjacent seagrass beds, thus in areas characterized by higher hydrodynamic disturbance than that experienced by the nearby mature meadows (Balestri et al., 2017 and the references therein). Suitable habitats for $P$. oceanica meadow colonization and survival could include sites characterized by high hydrodynamic exposure if substrates provided with proper lithological and topographical features are available.

There is a growing consensus that sexual reproduction, even at low recruitment rates, can confer long-lived clonal species an adaptive demographic advantage, since the increase in population genotypic diversity has been correlated to an increase in the capability to adapt to a changing environment and provides evolutionary potential to species (Reusch et al., 2005; Ehlers et al., 2008; Hughes and Stachowicz, 2011). Areas located near meadows that regularly flower and produce fruits, characterized by rocky substrates with adequate complexity, are promising candidates as successful sexual recruitment sites, even in highenergy hydrodynamic conditions. Locations with these features

\section{REFERENCES}

Alagna, A., Vega Fernández, T., D’Anna, G., Magliola, C., Mazzola, S., and Badalamenti, F. (2015). Assessing Posidonia oceanica seedling substrate preference: an experimental determination of seedling anchorage success in rocky vs. sandy substrates. PLoS One 10:e0125321. doi: 10.1371/journal.pone. 0125321

Alagna, A., Vega Fernández, T., Terlizzi, A., and Badalamenti, F. (2013). Influence of microhabitat on seedling survival and growth of the Mediterranean seagrass Posidonia oceanica (L.) delile. Estuar. Coast. Shelf Sci. 119, 119-125.

Alagna, A., Zenone, A., and Badalamenti, F. (2020). The perfect microsite: how to maximize Posidonia oceanica seedling settlement success for restoration purposes using ecological knowledge. Mar. Environ. Res. 161:104846. doi: 10. 1016/j.marenvres.2019.104846

Badalamenti, F., Alagna, A., and Fici, S. (2015). Evidences of adaptive traits to rocky substrates undermine paradigm of habitat preference of the Mediterranean seagrass Posidonia oceanica. Sci. Rep. 5:8804. doi: 10.1038/srep0 8804 should be included in conservation planning even if not currently covered by seagrass beds, in the view of facilitating future natural colonization processes as well as restoration initiatives based on the use of seeds and seedlings. Moreover, selection of shallow areas adjacent to existing seagrass beds should be considered in the face of sea level rise predicted for the next century and of the potential landward shifting of the ecosystems (Saunders et al., 2013; IPCC, 2019).

\section{DATA AVAILABILITY STATEMENT}

The raw data supporting the conclusions of this article will be made available by the authors, without undue reservation.

\section{AUTHOR CONTRIBUTIONS}

$\mathrm{AZ}, \mathrm{AA}$, and $\mathrm{FB}$ conceived and designed the research. AZ, EI, and $\mathrm{FB}$ conducted the experiments and analysis. EI contributed facilities and equipment. AZ, AA, and EI analyzed the data. $\mathrm{AZ}$ and $\mathrm{AA}$ wrote the manuscript. FB, EI, and SG revised the manuscript. All authors read and approved the final manuscript.

\section{FUNDING}

This study was supported by a travel grant provided by the Royal Swedish Academy of Sciences (KVA) and partially supported by the project Marine Hazard, PON03PE_00203_1, Italian Ministry of Education, University and Research (MIUR) and FORMAS grant Dnr. 2019-01192.

\section{ACKNOWLEDGMENTS}

We would like to thank FORMAS grant Dnr. 2019-01192, and Giuseppe Di Stefano for the technical support in rearing seeds at IAS-CNR Lab in Castellammare del Golfo.

Balestri, E., Piazzi, L., and Cinelli, F. (1998). Survival and growth of transplanted and natural seedlings of Posidonia oceanica (L.) delile in a damaged coastal area. J. Exp. Mar. Biol. Ecol. 228, 209-225.

Balestri, E., Vallerini, F., and Lardicci, C. (2017). Recruitment and patch establishment by seed in the seagrass Posidonia oceanica: importance and conservation implications. Front. Plant Sci. 8:1067. doi: 10.3389/fpls.2017. 01067

Belzunce, M., Navarro, R. M., and Rapoport, H. F. (2008). Posidonia oceanica seeds from drift origin: viability, germination and early plantlet development. Bot. Mar. 51, 1-9. doi: 10.1515/bot.2008.005

Borovec, O., and Vohník, M. (2018). Ontogenetic transition from specialized root hairs to specific root-fungus symbiosis in the dominant Mediterranean seagrass Posidonia oceanica. Sci. Rep. 8, 1-11. doi: 10.1038/s41598-018-28989-4

Boudouresque, C. F., Bernard, G., Bonhomme, P., Charbonnel, E., Diviacco, G., Meinesz, A., et al. (2012). Protection and Conservation of Posidonia oceanica Meadows. Tunis: RAMOGE and RAC/SPA publisher, 1-202.

Buia, M. C., Gambi, M. C., and Dappiano, M. (2004). Seagrass systems. Biol. Mar. Mediterr 10, 133-183. 
Castejón-Silvo, I., and Terrados, J. (2021). Poor success of seagrass Posidonia oceanica transplanting in a meadow disturbed by power line burial. Mar. Envir. Res. 170:105406. doi: 10.1016/j.marenvres.2021.105406

Dean, R. G., and Dalrymple, R. A. (1991). Water Wave Mechanics for Engineers and Scientist. Advanced Series on Ocean Engineering. Singapore: World Scientific. doi: $10.1142 / 1232$

Di Carlo, G., Badalamenti, F., and Terlizzi, A. (2007). Recruitment of Posidonia oceanica on rubble mounds: substratum effects on biomass partitioning and leaf morphology. Aquat. Bot. 87, 97-103.

Ehlers, A., Worm, B., and Reusch, T. B. H. (2008). Importance of genetic diversity in eelgrass Zostera marina for its resilience to global warming. Mar. Ecol. Prog. Ser. 355, 1-7. doi: 10.3354/meps07369

Guerrero-Meseguer, L., Sanz-Lázaro, C., and Marín, A. (2018). Understanding the sexual recruitment of one of the oldest and largest organisms on Earth, the seagrass Posidonia oceanica. PLoS One 13:e0207345. doi: 10.1371/journal.pone. 0207345

Hughes, R. A., and Stachowicz, J. J. (2011). Seagrass genotypic diversity increases disturbance response via complementarity and dominance. J. Ecol. 99, 445-453.

Infantes, E., de Smit, J. C., Tamarit, E., and Bouma, T. J. (2021). Making realistic wave climates in low-cost wave mesocosms: a new tool for experimental ecology and biogeomorphology. Limnol. Oceanogr. Meth. 19, 317-330.

Infantes, E., Orfila, A., Bouma, T. J., Simarro, G., and Terrados, J. (2011). Posidonia oceanica and Cymodocea nodosa seedling tolerance to wave exposure. Limnol. Oceanogr. 56, 2223-2232. doi: 10.4319/lo.2011.56.6.2223

Infantes, E., Terrados, J., Orfila, A., Cañellas, B., and Álvarez-Ellacuria, A. (2009). Wave energy and the upper depth limit distribution of Posidonia oceanica. Bot. Mar. 52, 419-427.

Inglis, G. J. (2000). Disturbance-related heterogeneity in the seed banks of a marine angiosperm. J. Ecol. 88, 88-99. doi: 10.1046/j.1365-2745.2000.00433.x

IPCC (2019). "Summary for policymakers," in IPCC Special Report on the Ocean and Cryosphere in a Changing Climate, eds D. C. Roberts, V. Masson-Delmotte, P. Zhai, M. Tignor, E. Poloczanska, K. Mintenbeck, et al.

Irving, A. D., Tanner, J. E., Seddon, S., Miller, D., Collings, G. J., Wear, R. J., et al. (2010). Testing alternate ecological approaches to seagrass rehabilitation: links to life-history traits. J. Appl. Ecol. 47, 1119-1127. i

Koch, E. W. (2001). Beyond light: physical, geological, and geochemical parameters as possible submersed aquatic vegetation habitat requirements. Estuaries 24, 1-17. doi: 10.2307/1352808

Koch, E. W., Ackerman, J. D., Verduin, J., and van Keulen, M. (2006). "Fluid dynamics in seagrass ecologyfrom molecules to ecosystems," in Seagrasses: Biology, Ecology and Conservation, eds A. W. D. Larkum, R. J. Orth, and C. M. Duarte (Dordrecht: Springer), 193-224. doi: 10.1007/978-1-4020-2983-7_8

Lasagna, R., Montefalcone, M., Albertelli, G., Corradi, N., Ferrari, M., Morri, C., et al. (2011). Much damage for little advantage: field studies and morphodynamic modelling highlight the environmental impact of an apparently minor coastal mismanagement. Estuar. Coast. Shelf Sci. 94, 255-262. doi: $10.1016 /$ j.ecss.2011.07.003

Marion, S. R., and Orth, R. J. (2012). Seedling establishment in eelgrass: seed burial effects on winter losses of developing seedlings. Mar. Ecol. Prog. Ser. 448, 197-207. doi: 10.3354/meps09612

Meysick, L., Infantes, E., and Boström, C. (2019). The influence of hydrodynamics and ecosystem engineers on eelgrass seed trapping. PLoS One 14:e0222020. doi: 10.1371 /journal.pone.0222020

Montefalcone, M. (2009). Ecosystem health assessment using the Mediterranean seagrass Posidonia oceanica: a review. Ecol. Indic. 9, 595-604. doi: 10.1016/j. ecolind.2008.09.013

Montefalcone, M., Vacchi, M., Carbone, C., Cabella, R., Schiaffino, C. F., Elter, F. M., et al. (2016). Seagrass on the rocks: Posidonia oceanica settled on shallowwater hard substrata withstands wave stress beyond predictions. Estuar. Coast. Shelf Sci. 180, 114-122. doi: 10.1016/j.ecss.2016.06.024

Olesen, B., Marba, N., Duarte, C. M., Savela, R. S., and Fortes, M. D. (2004). Recolonization dynamics in a mixed seagrass meadow: the role of clonal versus sexual processes. Estuaries 27, 770-780. doi: 10.1007/bf02912039

Pereda-Briones, L., Infantes, E., Orfila, A., Tomas, F., and Terrados, J. (2018). Dispersal of seagrass propagules: interaction between hydrodynamics and substratum type. Mar. Ecol. Prog. Ser. 593, 47-59. doi: 10.3354/meps12518
Pereda-Briones, L., Terrados, J., Agulles, M., and Tomas, F. (2020). Influence of biotic and abiotic factors of seagrass Posidonia oceanica recruitment: identifying suitable microsites. Mar. Environ. Res. 162:105076. doi: 10.1016/j.marenvres. 2020.105076

Piazzi, L., Acunto, S., and Cinelli, F. (1999). In situ survival and development of Posidonia oceanica (L.) delile seedlings. Aquat. Bot. 63, 103-112. doi: 10.1016/ s0304-3770(98)00115-6

Reusch, T. B., Ehlers, A., Hämmerli, A., and Worm, B. (2005). Ecosystem recovery after climatic extremes enhanced by genotypic diversity. Proc. Natl. Acad. Sci. U.S.A. 102, 2826-2831. doi: 10.1073/pnas.0500008102

Rivers, D. O., Kendrick, G. A., and Walker, D. I. (2011). Microsites play an important role for seedling survival in the seagrass Amphibolis antarctica. J. Exp. Mar. Biol. Ecol. 401, 29-35. doi: 10.1016/j.jembe.2011.03.005

Roca, G., Alcoverro, T., Krause-Jensen, D., Balsby, T. J. S., van Katwijk, M. M., Marbà, N., et al. (2016). Response of seagrass indicators to shifts in environmental stressors: a global review and management synthesis. Ecol. Indic. 63, 310-323. doi: 10.1016/j.ecolind.2015.12.007

Ruju, A., Ibba, A., Porta, M., Buosi, C., Passarella, M., and De Muro, S. (2018). The role of hydrodynamic forcing, sediment transport processes and bottom substratum in the shoreward development of Posidonia oceanica meadow. Estuar. Coast. Shelf Sci. 212, 63-72.

Saunders, M. I., Leon, J., Phinn, S. R., Callaghan, D. P., O’Brien, K. R., Roelfsema, C. M., et al. (2013). Coastal retreat and improved water quality mitigate losses of seagrass from sea level rise. Glob. Change Biol. 19, 2569-2583. doi: 10.1111/ gcb. 12218

Telesca, L., Belluscio, A., Criscoli, A., Ardizzone, G., Apostolaki, E. T., Fraschetti, S., et al. (2015). Seagrass meadows (Posidonia oceanica) distribution and trajectories of change. Sci. Rep. 5:12505. doi: 10.1038/srep12505

Vacchi, M., Montefalcone, M., Bianchi, C. N., Morri, C., and Ferrari, M. (2010). The influence of coastal dynamics on the upper limit of the Posidonia oceanica meadow. Mar. Ecol. 31, 546-554.

Vacchi, M., Montefalcone, M., Schiaffino, C. F., Parravicini, V., Bianchi, C. N., Morri, C., et al. (2014). Towards a predictive model to assess the natural position of the Posidonia oceanica seagrass meadows upper limit. Mar. pollut. Bull. 83, 458-466. doi: 10.1016/j.marpolbul.2013.09.038

Varenberg, M., Pugno, N., and Gorb, S. (2010). Spatulate structures in biological fibrillar adhesion. Soft Matter. 6:1. doi: 10.1007/978-3-319-42087-5_54-2

Zenone, A., Alagna, A., D’Anna, G., Kovalev, A., Kreitschitz, A., Badalamenti, F., et al. (2020a). Biological adhesion in seagrasses: the role of substrate roughness in Posidonia oceanica (L.) delile seedling anchorage via adhesive root hairs. Mar. Environ. Res. 160:105012. doi: 10.1016/j.marenvres.2020.105012

Zenone, A., Filippov, A. E., Kovalev, A., Badalamenti, F., and Gorb, S. N. (2020b). Root hair adhesion in Posidonia oceanica (L.) delile seedlings: a numerical modelling approach. Front. Mech. Eng. 6:590894. doi: 10.3389/fmech.2020. 590894

Conflict of Interest: The authors declare that the research was conducted in the absence of any commercial or financial relationships that could be construed as a potential conflict of interest.

The reviewer IM declared a past collaboration with one of the authors EI to the handling editor.

Publisher's Note: All claims expressed in this article are solely those of the authors and do not necessarily represent those of their affiliated organizations, or those of the publisher, the editors and the reviewers. Any product that may be evaluated in this article, or claim that may be made by its manufacturer, is not guaranteed or endorsed by the publisher.

Copyright (c) 2022 Zenone, Badalamenti, Alagna, Gorb and Infantes. This is an open-access article distributed under the terms of the Creative Commons Attribution License (CC BY). The use, distribution or reproduction in other forums is permitted, provided the original author(s) and the copyright owner(s) are credited and that the original publication in this journal is cited, in accordance with accepted academic practice. No use, distribution or reproduction is permitted which does not comply with these terms. 